\title{
Mass-Asymmetry effects in heavy ion reactions: Complete fu- sion Vs incomplete fusion
}

\author{
Sunil Dutt ${ }^{1}$, a, Avinash Agarwal ${ }^{1}$, Munish Kumar ${ }^{1}$, Vijay R. Sharma ${ }^{2}$, I. A. Rizvi², R. Kumar ${ }^{3}$, and \\ A. K. Chaubey ${ }^{4}$ \\ ${ }^{1}$ Department of Physics, Bareilly College, Bareilly, 243 005,INDIA \\ ${ }^{2}$ Department of Physics, Aligarh Muslim University, Aligarh 202002 INDIA \\ ${ }^{3}$ Nuclear Physics Group, Inter University Accelerator Centre, New Delhi 110067 INDIA \\ ${ }^{4}$ Department of Physics, Addis Ababa University, Addis Ababa- ETHIOPIA
}

\begin{abstract}
The excitation functions for ${ }^{59} \mathrm{Co}\left({ }^{12} \mathrm{C}, \mathrm{pn}\right){ }^{69} \mathrm{Ge},{ }^{59} \mathrm{Co}\left({ }^{12} \mathrm{C}, 2 \mathrm{pn}\right){ }^{68} \mathrm{Ga}$, ${ }^{59} \mathrm{Co}\left({ }^{12} \mathrm{C}, \mathrm{p} 4 \mathrm{n}\right){ }^{68} \mathrm{Ge},{ }^{59} \mathrm{Co}\left({ }^{12} \mathrm{C}, \alpha \mathrm{p} 4 \mathrm{n}\right){ }^{62} \mathrm{Zn}$ reactions for ${ }^{12} \mathrm{C}+{ }^{59} \mathrm{Co}$ projectile-target system have been measured for the first time. The recoil catcher technique followed by off-line $\gamma$-ray spectroscopy with HPGe detectors was used. The measured excitation functions are compared with the calculations based on the available statistical model codes. The probability of incomplete fusion is found to increase with the projectile energy. Moreover, an attempt has been made to observe the impact of mass-asymmetry on incomplete fusion probability. The effect of $\alpha$-break-up energy $\left(\mathrm{Q}_{\alpha}\right)$ on the ICF probability is also studied with the available data.
\end{abstract}

\section{Introduction}

Light heavy-ion $(\mathrm{Z} \leq 10)$ induced reactions, in the vicinity of $10 \mathrm{MeV} /$ nucleon projectile energies, has been the matter of treatise among experimental as well as theoretical nuclear physicists since the early sixties [1]. The study of heavy ion (HI) interactions is quite specific due to its complex structure and large momentum carried. HI interactions may be categorised in many ways, one of them is in terms of impact parameter. The direct reactions play an important role at higher values of impact parameter, leading to few nucleon transfer processes. However, at smaller values, complete fusion(CF), incomplete fusion (ICF)/ breakup fusion and deep-inelastic collision (DIC) processes may be governing [2]. At high energies, $>10 \mathrm{MeV} /$ nucleon, the concept of incomplete mass transfer in HI-induced reactions has originated after the pioneering experimental observation of 'fast- $\alpha$-particles' by Britt and Quinton [1]. Since then several studies have been carried out to study the ICF dynamics for projectile energies near $10 \mathrm{MeV} /$ nucleon. However, some recent studies in the last decade show the occurrence of incomplete fusion phenomenon at beam energies just above the Coulomb barrier [3-7]. Morgenstern et al. [8] in his studies established the entrance-channel mass-asymmetry dependence of the ICF reaction, with the ICF probability being higher in a mass-asymmetric system than in a mass-symmetric system at the same relative velocity. Later, studies by Vineyard et al. [9] and Chakrabarty et al. [10] also supported the findings of Morgenstern et al. [8].

\footnotetext{
a e-mail: sunilduttamu@gmail.com
} 
In this work, the excitation functions (EFs) for ${ }^{59} \mathrm{Co}\left({ }^{12} \mathrm{C}, \mathrm{pn}\right){ }^{69} \mathrm{Ge},{ }^{59} \mathrm{Co}\left({ }^{12} \mathrm{C}, 2 \mathrm{pn}\right){ }^{68} \mathrm{Ga}$, ${ }^{59} \mathrm{Co}\left({ }^{12} \mathrm{C}, \mathrm{p} 4 \mathrm{n}\right){ }^{66} \mathrm{Ge}$ and ${ }^{59} \mathrm{Co}\left({ }^{12} \mathrm{C}, \alpha \mathrm{p} 4 \mathrm{n}\right){ }^{62} \mathrm{Zn}$ reaction channels have been measured, which is an extension of our earlier work [3] on the measurement of EFs for ${ }^{59} \mathrm{Co}\left({ }^{12} \mathrm{C}, \mathrm{p} 3 \mathrm{n}\right){ }^{67} \mathrm{Ge}$, ${ }^{59} \mathrm{Co}\left({ }^{12} \mathrm{C}, 2 \mathrm{p} 2 \mathrm{n}\right){ }^{67} \mathrm{Ga}, \quad{ }^{59} \mathrm{Co}\left({ }^{12} \mathrm{C}, \alpha \mathrm{n}\right){ }^{66} \mathrm{Ga}, \quad{ }^{59} \mathrm{Co}\left({ }^{12} \mathrm{C}, \alpha 2 \mathrm{n}\right){ }^{65} \mathrm{Ga},{ }^{59} \mathrm{Co}\left({ }^{12} \mathrm{C}, \alpha \mathrm{p} 3 \mathrm{n}\right){ }^{63} \mathrm{Zn} \quad$ and ${ }^{59} \mathrm{Co}\left({ }^{12} \mathrm{C}, 2 \alpha 2 \mathrm{n}\right){ }^{61} \mathrm{Cu}$ reaction channels. An attempt has been made to study the dependence of ICF fraction on entrance-channel mass-asymmetry and $\mathrm{Q}_{\alpha}$-value for the available experimental data.
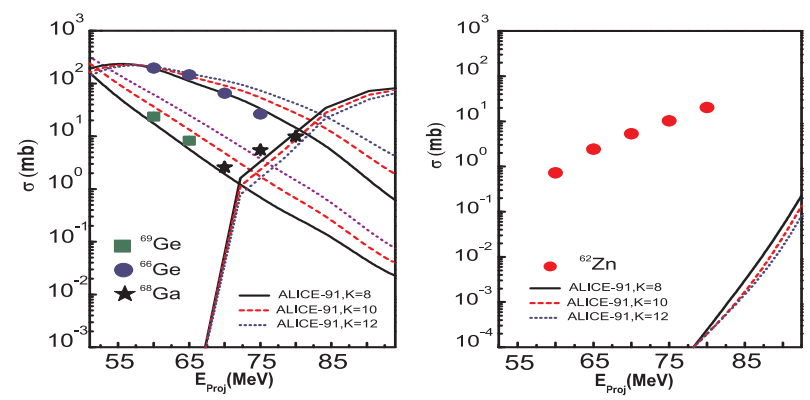

Figure 1. (color online) Experimental and theoretical excitation functions for the production of ${ }^{69} \mathrm{Ge},{ }^{66} \mathrm{Ge},{ }^{68} \mathrm{Ga}$, and ${ }^{62} \mathrm{Zn}$ isotopes.

\section{Experimental Details, Results and Discussion}

The experiment was performed using the General Purpose Scattering Chamber (GPSC) facility of Inter-University Accelerator Centre (IUAC), New Delhi (INDIA). The nuclear spectroscopic data used in the evaluation and measurement of cross-sections were adopted from the 'Table of Radioactive Isotopes' by Browne and Firestone [11]. The Evaporation residues produced from the considered reaction channels were identified by their characteristics $\gamma$-ray and decay curve analysis. The methodological details, experimental procedure, target preparation, data analysis etc. used in present work are similar to those as in our earlier publications [3, 4].

The theoretical analysis of the data was carried out using the statistical models based computer codes PACE-4 [12], CASCADE [13] and ALICE-91 [14, 15]. However Alice91 predictions were found to be in good agreement for complete fusion channels for the present target-projectile system [3]. Hence Alice91 predictions have been adopted for the present study. The detailed description on model codes and various input parameters optimized for the model calculations can be found in our earlier publications $[3,4]$.

It is well established that the enhancement of measured production cross-sections for the evaporation residues populated through $\alpha$-emitting channels over theoretically calculated values is mainly due to presence of ICF along with CF dynamics in heavy ion interactions above barrier energies [3-7]. In the present work the enhancement in the measured cross sections for the $\alpha$-emitting channels has been observed and concluded that these residues are not only populated via CF but ICF is also playing an important role, which is consistent with our earlier findings for the same projectile-target system [3]. The ICF contribution $\left(\sigma_{I C F}\right)$ for individual channels has been estimated by subtracting the theoretically calculated complete fusion cross sections by ALICE-91, from the experimentally measured cross sections. The total complete fusion cross section $\left(\sum \sigma_{C F}\right)$ was calculated by adding the experimentally observed cross-sections for all CF-evaporation residues for the present target - projectile system and the ALICE-91 predictions for the $\alpha$-emitting channels, at each projectile energy. The 
Table 1. Experimentally measured production cross-sections $\sigma(\mathrm{mb})$ of identified evaporation residues along with the $\sum \sigma_{C F}, \sum \sigma_{I C F}, \sigma_{T F}$, and $\mathrm{F}_{I C F}(\%)$. ' $^{\prime}$ ' Data adopted from our previous work [3].

\begin{tabular}{lllllllr}
\hline \hline $\mathrm{E}_{\text {Proj }}$ & $\begin{array}{l}{ }^{69} \mathrm{Ge} \\
(\mathrm{pn})\end{array}$ & $\begin{array}{l}{ }^{67} \mathrm{Ge}^{*} \\
(\mathrm{p} 3 \mathrm{n})\end{array}$ & $\begin{array}{l}{ }^{66} \mathrm{Ge} \\
(\mathrm{p} 4 \mathrm{n})\end{array}$ & $\begin{array}{l}{ }^{68} \mathrm{Ga} \\
(2 \mathrm{pn})\end{array}$ & $\begin{array}{l}{ }^{67} \mathrm{Ga}^{*} \\
(2 \mathrm{p} 2 \mathrm{n})\end{array}$ & $\begin{array}{l}{ }^{66} \mathrm{Ga}^{*} \\
(\alpha \mathrm{n})\end{array}$ & $\begin{array}{r}{ }^{65} \mathrm{Ga}^{*} \\
(\alpha 2 \mathrm{n})\end{array}$ \\
\hline 60 & $23.7 \pm 2.6$ & $77.4 \pm 5.2$ & - & $196.3 \pm 19.6$ & $356.4 \pm 0.9$ & $62.1 \pm 6.6$ & $134.8 \pm 6.1$ \\
65 & $8.21 \pm 1.1$ & $160.4 \pm 6.1$ & - & $146.2 \pm 14.6$ & $430.6 \pm 1.0$ & $72.0 \pm 6.0$ & $169.8 \pm 7.0$ \\
70 & - & $138.4 \pm 6.3$ & $2.6 \pm 0.3$ & $65.5 \pm 6.6$ & $542.9 \pm 1.0$ & $108.8 \pm 13.4$ & $98.9 \pm 6.9$ \\
75 & - & $178.9 \pm 9.7$ & $5.5 \pm 0.6$ & $26.5 \pm 2.7$ & $523.9 \pm 1.0$ & $214.6 \pm 17.9$ & $94.5 \pm 11.1$ \\
80 & - & $155.8 \pm 11.0$ & $10.0 \pm 1.0$ & - & $419.3 \pm 8.7$ & $473.2 \pm 25.4$ & $83.3 \pm 9.5$ \\
\hline $\mathrm{E}_{\text {Proj }}$ & ${ }^{63} \mathrm{Zn}^{*}$ & $62 \mathrm{Zn}$ & ${ }^{61} \mathrm{Cu}^{*}$ & $\sum \sigma_{C F}$ & $\sum \sigma_{I C F}$ & $\sigma_{T F}$ & $\mathrm{~F}_{I C F}(\%)$ \\
& $(\alpha \mathrm{p} 3 \mathrm{n})$ & $(\alpha \mathrm{p} 4 \mathrm{n})$ & $(2 \alpha 2 \mathrm{n})$ & & & & 14.41 \\
\hline 60 & - & $0.7 \pm 0.1$ & $28.1 \pm 2.5$ & 752.8 & 126.7 & 879.5 & 23.04 \\
65 & - & $2.4 \pm 0.5$ & $57.1 \pm 23.8$ & 805.2 & 241.1 & 1046.3 & 22.95 \\
70 & - & $5.3 \pm 0.7$ & $95.3 \pm 25.4$ & 815.3 & 242.8 & 1058.1 & 39.67 \\
75 & $180.4 \pm 16.7$ & $10.3 \pm 1.8$ & $218.1 \pm 3.2$ & 876.4 & 576.2 & 1452.6 & 56.26 \\
80 & $126.6 \pm 10.4$ & $20.3 \pm 2.3$ & $635.5 \pm 6.8$ & 841.5 & 1082.5 & 1924.0 & \\
\hline \hline
\end{tabular}

total incomplete fusion cross section $\left(\sum \sigma_{I C F}\right)$ was obtained by adding the incomplete fusion cross sections of all the measured evaporation residues populated through $\alpha$-emitting channels at each projectile energy. The total fusion cross section $\sigma_{T F}$ was obtained by simply adding $\sum \sigma_{C F}$ and $\sum \sigma_{I C F}$. The cross-sections for presently measured evaporation residues along with $\sum \sigma_{C F}, \sum \sigma_{I C F}, \sigma_{T F}$ and $\% \mathrm{~F}_{I C F}$ are presented in Table- 1 . The $\sum \sigma_{C F}, \sum \sigma_{I C F}$ and $\sigma_{T F}$ are plotted as a function of projectile energy and are shown in the fig-2(A). It can be seen from figure that as the projectile energy is increased, the ICF contribution increases with respect to the CF process. This may be implicit with the fact that, as the projectile energy increases, the probability of the breakup of incident projectile into $\alpha$ clusters (i.e., breakup of ${ }^{12} \mathrm{C}$ into $\alpha+{ }^{8} \mathrm{Be}$ ) increases. The value of $\mathrm{F}_{I C F}$, ie. the ratio of incomplete


Figure 2. (color online) (A) Total fusion cross-section $\sigma_{T F}$ along-with the sum of complete fusion cross-sections $\sum \sigma_{C F}$, and incomplete fusion cross-sections $\sum \sigma_{I C F}$ at different projectile energies for the system ${ }^{12} \mathrm{C}+{ }^{59} \mathrm{Co}$. (B) The probability of incomplete fusion $\left(\mathrm{F}_{I C F}\right)$ as a function of projectile energy. (C) The $\mathrm{F}_{I C F}$ as a function of entrance-channel mass-asymmetry at relative velocity $\left(\mathrm{v}_{r e l}=0.067 \mathrm{c}\right)$ for various systems. (D) The value of $\% \mathrm{~F}_{I C F}$ on same target have been plotted with $\mathrm{Q}_{\alpha}$-value. The lines are drawn to guide the eyes.

fusion cross section to the total fusion cross section $\left(\sum \sigma_{I C F} / \sigma_{T F}\right) 100 \%$ has been obtained and plotted 
as a function of projectile energy and is shown in fig 2(B). It is observed from the figure that the ICF fraction is consistently increasing with projectile energy. The ICF fractions for the present system ${ }^{12} \mathrm{C}+{ }^{59} \mathrm{Co}$ along with previously measured systems ${ }^{12} \mathrm{C}+{ }^{52} \mathrm{Cr}[5],{ }^{20} \mathrm{Ne}+{ }^{59} \mathrm{Co}$, and ${ }^{16} \mathrm{O}+{ }^{45} \mathrm{Sc}[7]$ as a function of mass-asymmetry for the same relative velocity $\mathrm{v}_{r e l}=0.067 \mathrm{c}$ have been calculated and displayed in fig-2(C), where $\mathrm{v}_{\text {rel }}$ is calculated by using the formula, as given in the ref [8]. The present measurements suggest that ICF probability increases with mass-asymmetry of the interacting partner and supports the findings of Morgenstern et al. [8]. To study the effect of projectile structure on incomplete fusion the probability of incomplete fusion, $\mathrm{F}_{I C F}$ has also been plotted against the $\mathrm{Q}_{\alpha^{-}}$ value of the projectiles for the same target and is shown in fig-2(D). From figure it is evident that as the $\mathrm{Q}_{\alpha}$-value of the projectile increases, the value of $\% \mathrm{~F}_{I C F}$ decreases. Hence it is worth mentioning that along with mass-asymmetry of interacting partners, the projectile structure (which predominantly depends on the $\mathrm{Q}_{\alpha}$-value of the projectile) also plays an important role in ICF reaction dynamics.

\section{Conclusions}

The large difference in our measured values over theoretical predictions gives clear signature of incomplete fusion for $(\mathrm{C}, \alpha \mathrm{p} 4 \mathrm{n})^{62} \mathrm{Zn}$ channel in the considered energy range. The analysis of the data also suggests that the projectile breakup probability leading to ICF, increases with projectile energy. It can also be seen that the ICF contributes significantly to the total fusion cross-sections, along-with the complete fusion above Coulomb barrier energies. The comparison of the present data with similar data on ${ }^{12} \mathrm{C}+{ }^{52} \mathrm{Cr}[5],{ }^{20} \mathrm{Ne}+{ }^{59} \mathrm{Co}$, and ${ }^{16} \mathrm{O}+{ }^{45} \mathrm{Sc}$ [7] systems suggest that ICF probability increases with mass-asymmetry of the interacting partners and follows Morgenstern's mass-asymmetry systematic. Moreover, we also found that with the increase of $\alpha$-Q-value $\left(\mathrm{Q}_{\alpha}\right)$, the incomplete fusion contribution to the total fusion decreases.

\section{Acknowledgments}

One of the author (S.D.) is thankful to UGC (India) for financial support through JRF in Major research Project Ref. F. No. 40-430/2011 (SR).

\section{References}

[1] H. C. Britt and A. R. Quinton, Phys. Rev. 124, 877 (1961)

[2] P. E. Hodgson, Nuclear Heavy Ion Reactions (Clarendon Press, Oxford, 1978)

[3] Avinash Agarwal et al., Int. J. Mod. Phys. E 17, 393 (2008) and references therein

[4] Kamal Kumar et al., Phys. Rev. C 87, 044608 (2013) and references therein

[5] F. K. Amanuel et al., Phys. Rev. C 84, 024614 (2011) and references therein

[6] Abhishek Yadav et al., Phys. Rev. C 85, 034614 (2012); Phys. Rev. C 86, 014603 (2012)

[7] D. Singh et al., Phys. Rev. C 83, 054604 (2011); J. Phys. Soc. Jpn. 75, 104201 (2006)

[8] H. Morgenstern et al. Phys. Rev. Lett. 52, 1104 (1984)

[9] M. F. Vineyard et al., Phys. Rev. C 45, 1784 (1992)

[10] S. Chakrabarty et al., Nucl. Phys. A 678, 355 (2000)

[11] E. Browne \& R. B. Firestone, Table of Radioactive Isotopes (John Wiley \& Sons, NY, 1986)

[12] A Gavron, Phys. Rev. C 21, 230 (1980)

[13] F. Puhlhofer, Nucl. Phys. A 280, 267 (1977)

[14] M. Blann, Phys. Rev. Lett. 27, 337 (1971); Phys. Rev. Lett. 28, 757 (1972)

[15] M. Blann, NEA Data Bank, Gif-sur- Y vette, France, Report PSR-146, (1991) 